$\xi=1$ 圆

\title{
An approach to mitigating the premature death of the battery of sensor node
}

\author{
Satyanarayana Chanagala ${ }^{1 *}$, Z.J.Khan ${ }^{2}$ \\ ${ }^{1}$ Research Scholar, RCERT, Department of Electronics and Power, Chandrapur \\ ${ }^{2}$ RCERT, Department of Electronics and Power, Chandrapur \\ *Corresponding author E-mail: scorppytek_satya@yahoo.com
}

\begin{abstract}
The prime objective of this paper is to investigate the impact of the sampling interval, which is one of the sensor node parameters, on the battery life of sensor node. An improper selection of sampling interval will lead to pronounced recovery effect which leads to premature death of the battery of sensor node. The suggested approach used in this work has achieved a significant lifetime improvement of the battery of sensor node.

Keywords: Battery; Discharge Efficiency; Lifetime; Sampling Interval; Sensor Node; Wireless Sensor Networks.
\end{abstract}

\section{Introduction}

A wireless sensor node has a limited power supply. A primary battery is used as the energy source of a sensor node of wireless sensor network deployed in a remote site. It is a fact that life of the battery decides the life of the sensor node. Therefore, the sensor node has to be discarded because of the restricted lifetime of the battery. Hence, there is a need to improve the lifespan of the battery. Therefore, in the extending of the lifetime of the WSNs, the battery plays a decisive role. Typically when the battery is exhausted, still it has a significant unused energy. This is due to unintelligent discharge of the battery. This early expiry of the battery is stated to as premature exhaustion of the battery. The rate and recovery phenomenon that occurs inside the battery, result in its early death. The technique suggested in this work diminishes the recovery effect, which in turn improves the capacity of the battery of sensor node.

\section{Present methodologies for extending the lifetime of wireless sensor networks}

Energy savings of considerable level have been accomplished by non-intersecting time operation of sets of sensor nodes [1,28].

Reasonable improvement in the lifetime has been achieved by reducing the power for transmitting the data and rising the duration of the transmission [2].

In Surveillance system using sensor systems, long lifetime is vital The lifetime in such applications is prolonged [3] in systematic steps.

A set of algorithms based on set covers has been designed [4] with which a good level of life improvement has been recorded.

Lengthening the operating lifespan of the wireless sensor network has been achieved by the controlled sink movements [5]. In the investigation, authors have found that the sensor nodes that communicate with the sink node incline to drain their energy quicker than others. Due to this, the sink gets inaccessible from the rest of the network, although most of the other nodes are functioning.
Collection of data and eliminating redundancy combined with optimum routing has resulted in increasing the sensor network lifetime [6].

A technique has been reported in [7], [28] for analyzing the null energy zones in the sensor field. In this method, the sensors are strategically deployed in the form of nonintersecting concentric sets. A model with a distance assignment approach was implemented to increase the operational time of wireless networks.

Wireless sensor networks have unlocked different fields to scattered data acquisition. There are fair chances that such networks are prone to early failure due to depletion of their batteries because of workload disparities and dissimilar hardware. This problem was mitigated by a different battery allocation formulation [8], [28].

The increase in the operating life of wireless sensor networks can be achieved if transmission of data is not time critical. As an alternative, the node can store the data and send the same when the most favorable location of sink node is found [10], where an arrangement that takes time constraints into consideration has been designed. Encouraging results have been observed this approach.

Topographies which are impassable and unfriendly regions, one of the ways to get the intelligence about the different physical quantities is by deploying an enormous number of sensors. Lifetime extension becomes important in such conditions. To increase the sensor network lifetime, a few power saving approaches were suggested [11], [28]. Authors, in their work, used a two-tiered wireless sensor network. Here, nodes are split into groups and nodes forward data to base stations via their heads. A wireless sensor system that uses approach of inquiry has been investigated [12] in which a user would issue a request and anticipate a response within a time bound frame.

Increasing the lifetime of dissimilar wireless sensor networks occurred at a sluggish pace. Motivated by ants, and experimental information, an approach has been formulated [13], [28]. Based on this information, an optimal path on the construction graph is constructed to increase the number of coupled covers. This approach is used to reflect the interest of device tasks. This technique has resulted in the extension of the lifetime of heterogeneous WSNs. 
The considerable level of lifetime improvement has been achieved with multiple sinks which have the ability of locomotion $[14,28]$. In time-critical applications, delay can't be tolerated and it is necessary to find the shortest path tree to ensure the long lifetime. A model based on mathematics has been formulated [15, 28] which has ensued in enhancement in the network lifetime with the deployment of the increased number of nodes.

Random allocation of data to the sensor nodes could be a reason for the early death of the sensor networks. Alleviating this problem has improved the lifespan of the wireless sensor networks [16, 28]. In this approach, the authors presented distinct nodes known as the mobile agent. Authors have considered a technique based on energy forecast which enabled these special nodes to identify approximately the residual energy in their cluster comprising of other sensors. This approach has resulted in circumventing the problem of uneven consumption of energy.

In some wireless sensor applications, there could be a scenario where various regions involve dissimilar levels of sensing [17]. To address this bottleneck, authors have proposed a multi-objective sleep-scheduling scheme for differential coverage in wireless sensor networks to attain a better trade-off between coverage, lifespan, and energy consumption.

The wireless sensor networks applications where there are no time constraints; the energy consumption can be minimized with a mobile sink that could result in extending the lifespan of the wireless sensor network. Work in this direction has been undertaken with significant improvements in the lifetime of the networks [18]. The depletion of nodes energy results in the death of the wireless sensor network. The sensor network will function even when one sensor node or few nodes are exhausted, till the sensor nodes data reaches the sink node. Thus, the wireless sensor network lifetime takes the lifespan of all sensors into consideration that generate the valuable data of the physical phenomenon. Work in this direction has been carried out [19] with reasonable gains in the lifetime of the sensor network.

In the case of the end-to-end delivery delay constraints [20, 28], the challenge of lifetime improvement of the WSNs, a methodology named the coordination of the intra-node has been investigated. One of the approaches to enhance the wireless sensor network longevity is to build a work-sharing forwarding tree structure placed at the data collecting sink node. But, this could result in a long path while communicating with the sink node. It is unfavorable for some time-critical applications as such applications require that all the data of sensed physical phenomenon required by the sink node. This situation may not suit some mission-critical applications that require all sensed data to be received by the base station with the smallest lag. An investigation is carried out in this direction [21] with positive results.

By adding redundant nodes, it is likely to enhance the operational time of the sensor network [22], [28]. These nodes can be activated at the times when any active node exhausted of its energy. Authors have adopted the above-discussed strategy by proposing the Low-Energy Adaptive Clustering Hierarchy spare management protocol.

Maximum energy consumption of the WSN is due to the communication unit. Hence, it requires a good deal of supervision to mitigate the problem of the early death of the nodes in the close proximity of the sink node. The network lifetime depends on the reliability of the communication path and energy constraints of the nodes in the close proximity of the sink node. These two problems have been addressed [23] with reasonable gains in the networks lifetime.

Optimum performance of the battery of sensor node has been achieved for sampling interval less than the optimum value [24], [28] by populating the sensor field with more nodes.

Randomness that exists in the clustering algorithms results in cluster heads more than the required. Investigation in this direction has been undertaken [25] with an approach to control the randomness present in LEACH's clustering algorithm.

Experimental results [26] have shown that the unwanted cluster heads lead to early exhaustion of the sensor network. To mitigate this problem, authors have adopted the dynamic cluster head selection method for a wireless sensor network.

A work to increase the reliable data communication to save the energy of the wireless sensor network has been undertaken [27], where the authors have suggested a Grid-Based Reliable Routing technique.

Rate capacity of the battery of sensor node results in the early discharge of the battery. A technique to counter the above effect has been suggested with the positive results [28].

Network lifetime and information reliability are two important aspects of wireless sensor networks. The contribution by authors [29] in this area has resulted in considerable improvement in the lifetime and reliability of the WSN.

In some cases the uneven distribution of data among the nodes of the WSN may result in shortening of the network lifetime. The problem is more pronounced in unplanned networks. A strategy that overcomes the above-discussed problem has been proposed [30] with significant improvements over existing schemes.

\section{Suggested methodology}

The work presented in this paper has considered recovery effect of the battery. Rate and recovery phenomenon are two important characteristics of the battery, which influences its lifetime.

The load connected to the battery result in movement of positively charged ions that are expended at the cathode-electrolyte boundary and are replaced by anode with new ions that flow through the electrolyte. When the connected load is high, the rapidity of generation of ions becomes insufficient as compared to the rapidity at which the cathode consumes them. This results in a net decrease of cations at the cathode and increase of the same at the anode, decreasing the output voltage of the battery. This process is referred to as the recovery effect.

The concentration gradient decreases if the battery is left idle for a period of time, which results in an apparent recovery of charge. This helps in the increase in the lifetime of the battery.

If it is possible to find this idle time during which the battery recovers, by making this value of idle time equal to the sensor node's sampling interval, there will be a definite possibility of prolonging the sensor node lifetime. This value of sampling interval for which an enlargement in the lifetime of the battery of sensor node is achieved is defined as optimum sampling interval.

An algorithm is designed to find the optimum sampling interval which is shown in the Fig.1.

\section{Experimental setup}

The sensor nodes used in the experiment are designed with each sensor node equipped with light, accelerometer, and temperature sensors. Each sensor node is controlled by the microcontroller and the sensed signals are conditioned by LM324IC. The microcontroller used here is PIC18F252. 

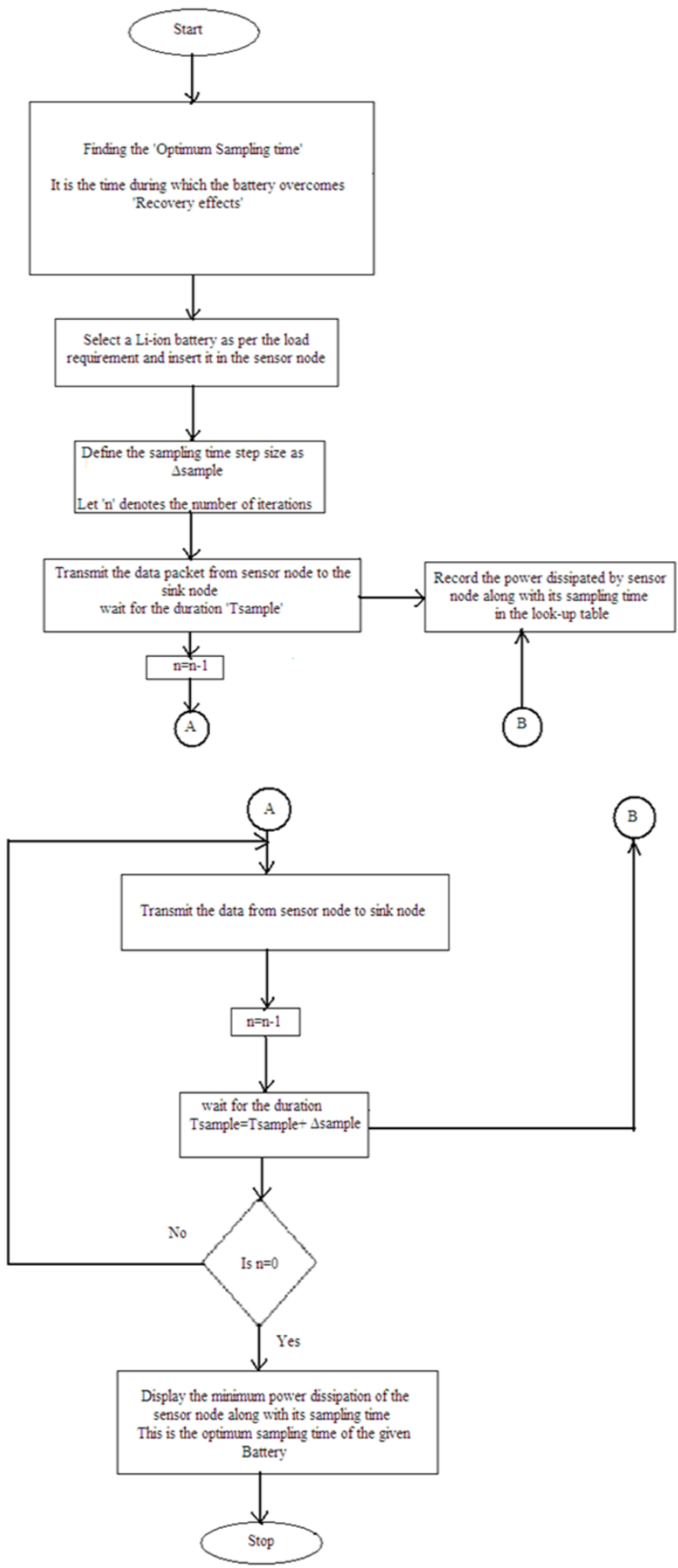

Fig. 1: Schematic of A Typical Sensor Node.

This microcontroller comes with high-end flash memory and has a 10-bit ADC. Analog physical phenomenon sensed by the sensors is converted into digital form by the on-chip analog to digital converter. In the experiment, the smallest detectable voltage is set at $4.88 \mathrm{mv}$. The baud rate of 9600 is used by on-chip USART of the microcontroller. The sampling interval is adjusted by using Timer ' 1 ' of the microcontroller. The microcontroller is operated by a $20 \mathrm{MHz}$ crystal oscillator. Master node or sink node is designed to facilitate the adjustment of various parameters like sampling interval, baud rate, and power required to transmit the data. Each sensed analog physical phenomenon is converted into digital twobyte data. Therefore six bytes of digital data is generated corresponding to the three sensors. In addition to this, a pair of data bytes is used for tracing the power consumed by the node. Further, one start bit and one stop bit which occupies two-byte length are used for facilitating smoother communication among the nodes. And an additional nine number of bytes are used to provide data security. Thus in total ten bytes are sent to the sink node nine times during the duration of the sampling interval to make the intermodal communication of better quality. Thus, finally, ninetynine bytes are sent by the sensor node to the sink node. The distance among the nodes is maintained at hundred meters.

Lithium-ion batteries are used to energize the nodes. Three such batteries with $2200 \mathrm{mAh}, 3.7 \mathrm{~V}$ ratings are used. A constant voltage of five volts is ensured to source microcontroller with regulated five volts using 7805 IC regulator via IC2941 to better the supply efficiency of IC regulator and this output is finally fed to the microcontroller. Texas Instrument's CC2500 is used to facilitate the intermodal communication link. The CC2500 is an attractive choice for very low-power communication uses. The transceiver is equipped with a highly adaptable baseband modem. The modulator-demodulator unit provides various modulation setups and supports data rate up to $500 \mathrm{KBaud}$. Further, CC2500 offers wide hardware provision for data packet handling, data safeguarding, burst transmissions, assessment of clear channel, an indication of link quality, and activate-on-radio. It requires +3.3 volts supply. It operates at $2.4 \mathrm{GHz}$. The circuit is designed for the $2400-2483.5$ MHz ISM (Industrial, Scientific, and Medical) frequency band.

Sink node also consists of CC2500 along with the microcontroller to collect the data bytes sent by the sensor node and it is programmed as a master node to change the parameters of sensor node as necessary by the experimentation. Further, the output of the sink node is interfaced to the computer to trace the experimental results. The experimental arrangement is as shown in the Fig.3.

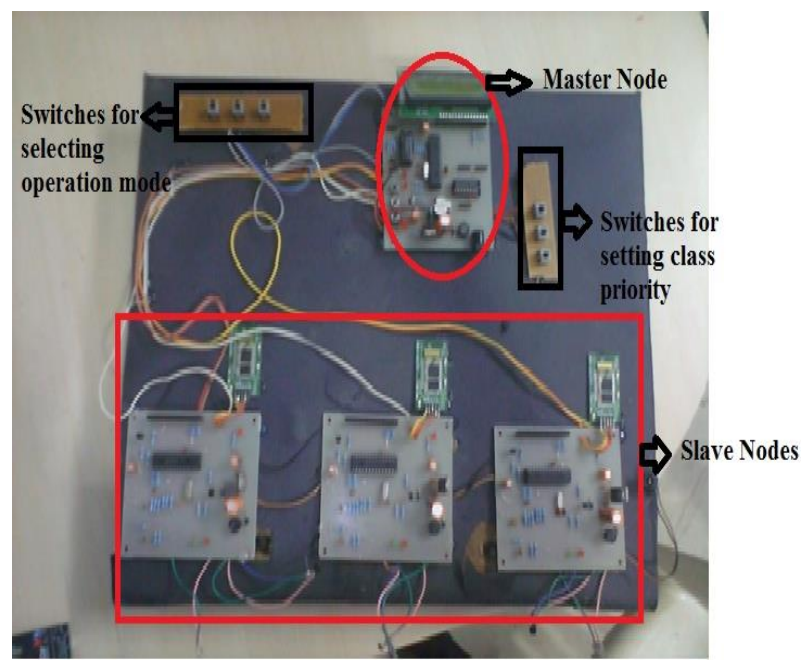

Fig. 2: Fabricated Sensor Nodes Arrangement.

\section{Results and discussions}

Inter nodal wireless communication is established by the RF communication module present on the nodes. The sink node or master node receives the data sent by the sensor node and in turn, sink node sends the same to the computer node which is interfaced to it. The microcontroller present on the master node is configured to trace the power consumed by the slave node. Further, the microcontroller on the slave node is programmed to increment the sampling interval with 10 milliseconds as the incremental value. The initial value of the sampling interval is chosen as 0.2 seconds. Also, the microcontroller on the master node is programmed to facilitate to trace power used by the sensor node at each sampling interval along with the lifetime of the node. Above discussed procedure is carried out for two hundred different sampling intervals. Along with the tracing of power dissipation and lifetime of the sensor node, one more facility is embedded into the controller program to trace the number of bytes received by the sink node. Experimental results are shown in the Fig.3. Power dissipation of 0.78 watts is recorded when the sampling interval of 0.2 seconds. This is the maximum value for power dissipation amongst all the 
sampling intervals. At this sampling interval, the battery discharges more rapidly due to pronounced recovery effect. With progressive increments of the sampling intervals, the power used by the sensor node shows a decreasing trend. However, when the sampling interval reaches 0.62 seconds, the lowest power dissipation of 0.35 watts is observed.

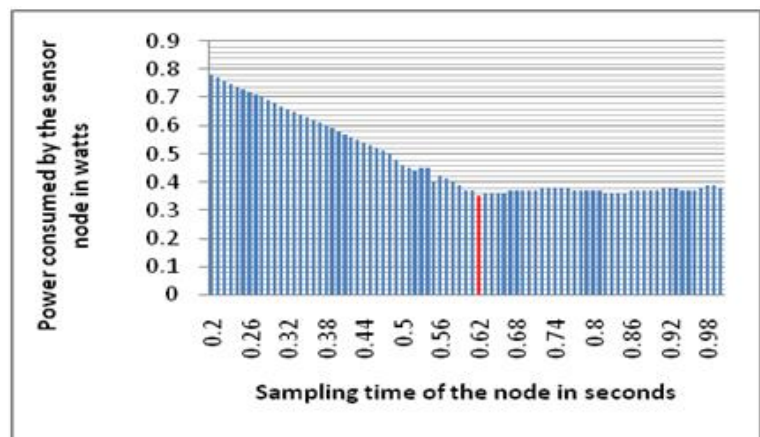

Fig. 3: Sampling Interval Versus Power Dissipated by the Sensor Node.

To validate the optimum choice of sampling interval obtained in the above experiment another experiment is undertaken. In this experiment, instead of choosing sampling intervals sequentially, they have been considered randomly. At each randomly selected valve of the sampling interval, the power used by the sensor node is traced. However, once again the minimum power dissipation is recorded at 0.62 seconds. Observed experimental results are displayed in Fig.4.

Another experiment has been conducted to assess the quality of service at optimum sampling interval.

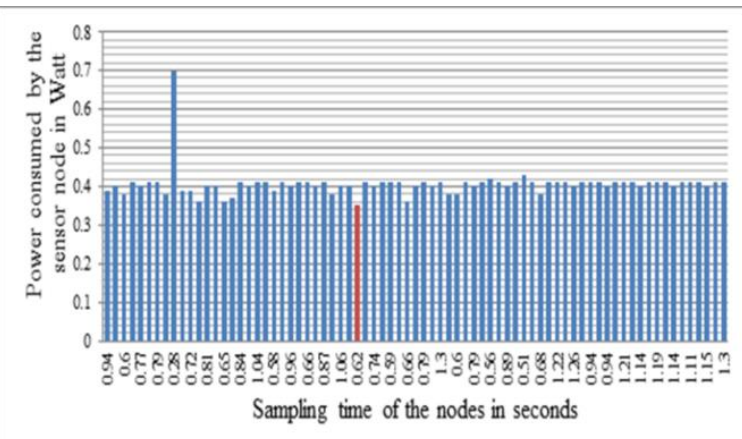

Fig. 4: Sampling Interval Versus Power Dissipated by the Sensor Node

Here, the sensor node is made to transmit the sensed data bytes for seventy-five number of times at the sampling interval of 0.62 seconds. In each attempt, the received number of data bytes are compared with that of transmitted. If the received number of data bytes are same as that of sent data bytes from the sensor node, it indicates an excellent quality of service.

Fig. 5 shows the results of the experiment. The mean value of bytes received by the sink node in seventy-five attempts is 94 out of 99 bytes being transmitted by the slave node. this effectively results in $95.36 \%$ of successful data byte reception. And this value of above $95 \%$ reflects a fairly better quality of service.

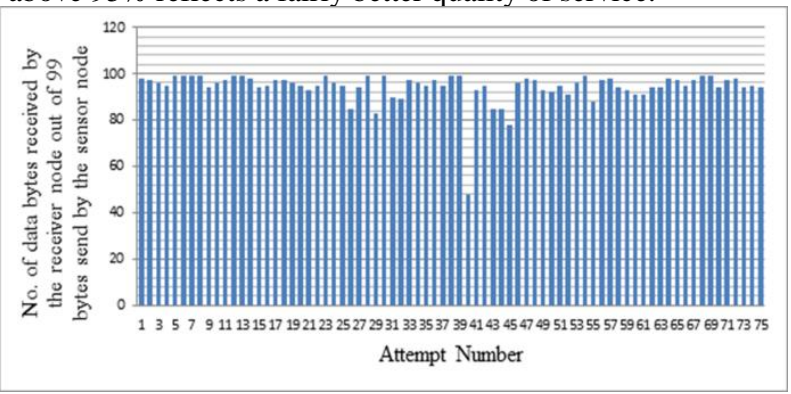

Fig. 5: Graph Shows the Quality of Service at the Optimum Sampling Interval.
A final experiment is arranged to find out the lifetime of the battery of sensor node at the progressively increasing value of the sampling intervals. Fig. 6 highlights the results. From the results, it is evident that the sensor node exhibits maximum lifetime at the optimum sampling interval of 0.62 seconds.

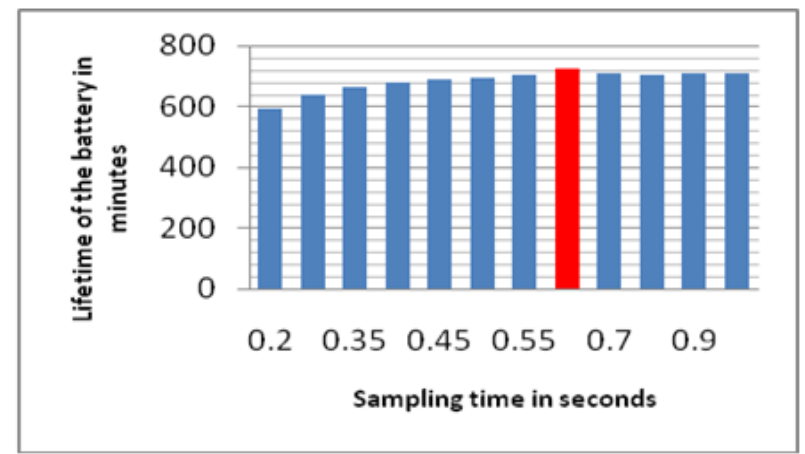

Fig. 6: Lifetime of the Battery Versus Sampling Interval.

Parameter improvement with the suggested technique is illustrated in Table 1. Here a comparative performance is presented when the chosen sampling interval is optimum and when the chosen sampling interval is arbitrary.

Table 1: Comparative Performance at Optimum and Arbitrary Sampling Intervals of the Sensor Node

\begin{tabular}{llll}
\hline $\begin{array}{l}\text { Sampling } \\
\text { interval }\end{array}$ & $\begin{array}{l}\text { Optimum } \\
\text { value } 0.62 \\
\text { seconds }\end{array}$ & $\begin{array}{l}\text { Arbitrary } \\
\text { value } 0.2 \\
\text { seconds }\end{array}$ & $\begin{array}{l}\text { \% Improvement } \\
\text { with optimum } \\
\text { value }\end{array}$ \\
\hline $\begin{array}{l}\text { Power } \\
\text { dissipated by } \\
\text { the sensor node }\end{array}$ & 0.35 watts & 0.78 watts & 55 \\
$\begin{array}{l}\text { Quality of } \\
\text { service }\end{array}$ & $96 \%$ & $62 \%$ & 34 \\
$\begin{array}{l}\text { The lifetime of } \\
\text { the sensor node }\end{array}$ & 722 Minutes & 590 Minutes & 18 \\
\hline
\end{tabular}

\section{Conclusion}

The methodology used in this work has mitigated the recovery effect of the battery. Therefore, it is suggested to operate the sensor node with the optimum sampling interval. An arbitrary choice of the same without understanding the impact of recovery effect of the battery of the sensor node may culminate in the early death of the battery.

\section{References}

[1] M. Cardei and D. Du. (2005), "Improving wireless sensor network lifetime through power aware organization," ACM Wireless Networks, volume11.

[2] Yang Yu, Victor Prasanna, and Bhaskar Krishnamacharya. (2006), "Energy Minimization for Real-Time data gathering in wireless sensor Networks," IEEE Transactions on wireless communications, volume 5, No.11.

[3] Hai Liu, Xiaohua Jia, Peng-Jun Wan, Chih-Wei Yi, S.Kami Makki, and Niki pissinou.(2007), "Maximizing Lifetime of sensor surveillance systems," IEEE/ACM Transactions on Networking, volume 15, Number 2. https://doi.org/10.1109/TNET.2007.892883.

[4] Suhil K.Prasad, Akshaya Dhawan, (2007), "Distributed Algorithms for the lifetime of wireless sensor networks based on dependencies among cover sets," Springer Berlin Heidelberg.

[5] Stefano Basagni, Alessio carsi, Emanuel Melachrinoudis, Chiara Petrioli, Z.Maria wang. (2008) "Controlled sink mobility for prolonging wireless sensor networks lifetime," Springer Science +Business Media, LLC 2007, Wireless network, volume 14, pp. 831_858.

[6] Cunqing Hua and Tak-Shing Peter Yun, (2008), "Optimal Routing and Data Aggregation for Maximizing lifetime of wireless sensor Networks, IEEE/ACM Transactions on Networking," Volume 16, Number 4 
[7] Chao Song, Ming Liu, Jiannong Cao, Yuan Zheng, Haigang Gong, and Guihai Chen,(2009) "Maximizing network lifetime based on transmission range adjustment in wireless sensor networks," Computer Communications- The International Journal for the Computer and Telecommunications industry. Elsevier, pp. 1316-1325. https://doi.org/10.1016/j.comcom.2009.02.002.

[8] HenguLong, YongpanLiu, Yiqun Wang, Robert P.Dick, and Huazhong Yong, (2009), "Battery allocation for wireless sensor network lifetime maximization under cost constraints", ICCAD-09, Sanjose, California, USA.

[9] Kobi Cohen and Amir Leshen, (2010), “A Time-varying opportunistic approach to lifetime maximization of wireless sensor networks," IEEE Transactions on signal processing, Volume 58, Number 10, pp. 5307-5319. https://doi.org/10.1109/TSP.2010.2052459.

[10] Youngsang Yun, Xia Y,(2010), "Maximizing the lifetime of wireless sensor networks with mobile sink in Delay-Tolerant applications," IEEE Transactions on Mobile Computing, Volume 9, issue 9, pp. $1308-1318$

[11] Ines Slama, Bodii Jouaber, Djamal Zeghlache, (2010), "Topology control and Routing in large-scale wireless sensor networks," Scientific Research, wireless sensor network, pp. 584-598. https://doi.org/10.4236/wsn.2010.28070.

[12] Ing-Ray Chen, AnhPhan Speer, Mohamed Eltoweissy.(2011), "Adaptive fault-tolerant QoS Control Algorithms for Maximizing system lifetime of Query-based wireless sensor networks," IEEE Transactions on Dependable and secure computing, Volume 8 , Number 2.

[13] Ying Lin, Jun Zhang, Henry Shu-Hung Chung, Wai Huang Ip, Yun Li, and Yu-Hui Shi".(2011),"Ant Colony optimization approach for maximizing the lifetime of Heterogeneous wireless sensor networks," IEEE Transactions on Systems, MAN, and Cybernetics Part C: Applications and Reviews, 42(3), pp. 408-420. https://doi.org/10.1109/TSMCC.2011.2129570.

[14] Stefano Basagni, Alessio Carosi, Chiaro petrioli, Cynthia A. Phillips, (2011), "Coordinated and controlled mobility of multiple sinks for maximizing the lifetime of wireless sensor networks," ACM Volume 17, issue 3, pp.759-778. https://doi.org/10.1007/s11276010-0313-8.

[15] Dijun Luo, Xiaojun, Xiaobing Wu, Guihai Chen, (2011),"Maximizing lifetime for shortest path aggregation Tree in wireless sensor networks," IEEE INFOCOM, pp: 1566-1574.

[16] Kai Lin, Min Chen, Sherali Zeodally, Joel J. P. C. Rorigues, (2011),"Balancing energy consumption with mobile agents in wireless sensor networks, " The International Journal of Grid Computing and science-Elsevier, Volume 28, Issue 2, pp. 446-456.

[17] Sengupta s. Das, Nasir M, (2012),"An evolutionary multiobjective sleep-scheduling scheme for differentiable coverage in wireless sensor networks," IEEE Transactions on Systems, MAN, and Cybernetics, Part C: Applications and Reviews, pp. 1093-1102.

[18] Zichan Xu, Weifa Liang, Yinlong Xu,(2012),"Network Lifetime maximization in delay-tolerant sensor networks with a mobile sink," 8th IEEE International conference on distributed computing in sensor systems, pp: 09-16.

[19] Liang Zhang, Shigang Chan, Ying Jian, Yuguang Fang, Zhen Mo.(2013),"Maximizing lifetime vector in wireless sensor networks," IEEE/ACM Transactions on Networking, Volume 21, Number $\quad 4, \quad$ pp. 1187-1200. https://doi.org/10.1109/TNET.2012.2227063.

[20] Ying Peng, Zi Li, Daji Qiao, Wensheng Zhang, (2013), “IC2: A Holistic approach to prolong the sensor network lifetime," IEEE INFOCOM, Turin, Italy, April 14-19.

[21] Feng Shan, Weifa Liang, Jun Luo, Xiaojun Shen, (2013), "Network lifetime maximization for time-sensitive data gathering in wireless sensor networks," Computer Networks 57 Elsevier, pp. 1063-1077. https://doi.org/10.1016/i.comnet.2012.12.005.

[22] Bilal Abu Bakr, Leszek T. Lilien, (2014), "Extending lifetime of wireless sensor networks by the management of spare nodes," The second international workshop on communications and sensor networks-Comsense-2014, Procedia computer science 34, Science Direct, Elsevier, pp. 493-498.

[23] Ali Ghaffari, (2014), "An energy-efficient routing protocol for wireless sensor networks using A-star algorithm," Journal of applied research and technology, Volume 12, Elsevier, pp. 815-822, August 2014.

[24] Satyanarayana C. and Zafar Jawed Khan,(2016),"An approach to maximize the performance of wireless sensor network for sampling time less than the optimum value", Fifth International Conference on Research Methodologies in Electronic Devices and Circuits EDC 2016, pp. 51-56.
[25] P. K. Batra and K. Kant, "LEACH-MAC: a new cluster head selection algorithm for Wireless Sensor Networks," Wireless Networks, vol. 22, no. 1, 2016, pp.49-60. https://doi.org/10.1007/s11276-0150951-y.

[26] D. Jia, H. Zhu, S. Zou, and P. Hu, "Dynamic cluster head selection method for the wireless sensor network," IEEE Sensors Journal, vol. 16, no. 8, 2016, pp. 2746-2754. https://doi.org/10.1109/JSEN.2015.2512322.

[27] X. Meng, X. Shi, Z. Wang, S. Wu, and C. Li, "A grid-based reliable routing protocol for wireless sensor networks with randomly distributed clusters," Ad Hoc Networks, vol. 51, 2016, pp. 47-61. https://doi.org/10.1016/j.adhoc.2016.08.004.

[28] Zafar Jawed Khan and Satyanarayana Chanagala, "A Transmission Power level aware approach to maximize the Lifetime of Wireless Sensor Network", WJERT, 2017, Volume 3, Issue 5, pp.336-350.

[29] Elma Zanaj, Indrit Enesi, Blerina Zanaj, "Energy-aware algorithms for Wireless Sensor Networks", Vol. 105, 2017, Elsevier, pp. 105 110.

[30] K. P. Sharma and T. P. Sharma, "Energy-hole avoidance and lifetime enhancement of a WSN through load factor," Turkish Journal of Electrical Engineering \& Computer Sciences, vol. 25, no. 2, 2017, pp. 1375-1387. https://doi.org/10.3906/elk-1508-162. 